\title{
温和条件下钉/氮掺杂碳纳米笼的苯乙酮选择性催化加氢性能
}

\author{
蔡跃进 ${ }^{\dagger}$ 刘晨霞 ${ }^{\dagger}$ 卓欧吴强 ${ }^{*}$ 杨立军陈强 \\ 王喜章 ${ }^{*}$ 胡征
}

(南京大学化学化工学院 介观化学教育部重点实验室 南京 210023)

\begin{abstract}
摘要 共轭羰基化合物的羰基选择性加氢反应被广泛用于制备重要的药物和化学中间体. 利用氮掺杂碳纳米笼 (hNCNC)大的比表面积和掺杂氮原子的针定作用, 构建了 $10 \mathrm{wt} \% \mathrm{Ru}$ 负载量的 Ru/hNCNC 催化剂, 尺寸约 $2.4 \mathrm{~nm}$ 的 Ru 纳米颗粒高度均匀地分散在 $\mathrm{hNCNC}$ 表面. 用于催化苯乙酮选择性加氢制 1-苯乙醇, 在 $50.0{ }^{\circ} \mathrm{C} 、 2.0 \mathrm{MPa} \mathrm{H}_{2}$ 的温和条 件下，展现出优异的催化加氢性能: 反应 $2.0 \mathrm{~h}$ 后的苯乙酮转化率和 1-苯乙醇选择性分别达到 $96.2 \%$ 和 $95.8 \%$, 远优于 未掺杂碳纳米笼 $(\mathrm{hCNC}$ ) 和活性炭负载的 Ru 催化剂; 循环使用 6 次后, 其苯乙酮转化率仅略有下降(从 $96.2 \%$ 到 $94.0 \%$ ), 明显优于 $\mathrm{Ru} / \mathrm{hCNC}$. Ru/hNCNC 的优异催化性能可归因于: hNCNC 大的比表面积和掺杂氮原子的针定作用有利于 $\mathrm{Ru}$ 纳米粒子的分散和固载、独特的微孔一介孔-大孔共存的分级孔结构有利于传质、掺杂氮原子有效调变了 $\mathrm{Ru}$ 催化剂的 电子结构.
\end{abstract}

关键词 苯乙酮; 选择性加氢; 1-苯乙醇; 氮掺杂碳纳米笼; 钉

\section{Ruthenium Nanoparticles Supported on Hierarchical Nitrogen-Doped Carbon Nanocages for Selective Hydrogenation of Acetophenone in Mild Conditions}

\author{
Cai, Yuejin ${ }^{\dagger}$ \\ Liu, Chenxia ${ }^{\dagger}$ \\ Zhuo, Ou \\ Wu, Qiang* \\ Yang, Lijun \\ Chen, Qiang
}

Wang, Xizhang*

$\mathrm{Hu}$, Zheng

(Key Laboratory of Mesoscopic Chemistry of MOE, School of Chemistry and Chemical Engineering, Nanjing University, Nanjing 210023)

\begin{abstract}
The selective hydrogenation of carbonyl groups of the conjugated carbonyl compounds is an important reaction in the pharmaceutical and chemical industries, and several selective hydrogenation approaches have been developed. Using stoichiometric hydrides $\left(\mathrm{LiAlH}_{4}, \mathrm{NaBH}_{4}\right.$, etc. $)$ as hydrogenation reagents has some shortcomings, including the unsatisfied selectivity of target product owing to the simultaneous hydrogenation of conjugated double bonds and carbonyl groups, as well as the flammability and explosibility of hydrides. Hydrogen is an alternative hydrogenation reagent, which can selectively hydrogenate carbonyl groups by homogeneous and heterogeneous catalytic processes. The noble metal ( $\mathrm{Ru}, \mathrm{Pd}$, etc.) complexes were usually used in the homogeneous catalytic process, which caused some serious issues such as the metal residues in products and the difficulties of recovering precious catalysts. These problems can be effectively solved by the heterogeneous catalytic process using the supported catalysts. Carbon-based materials, metal oxides and $\beta$-Zeolite are commonly used supports. Among them, carbon-based materials are preferable due to their features of abundant morphologies and structures, good stability, adjustable specific surface areas and pore structures, easy doping, etc. Interestingly, the introduction of heteroatoms into carbon matrix can provide a plenty of anchoring sites to disperse catalytically active species and regulate the interaction between active species and support, and hence promotes their catalytic properties. In addition, the high specific surface areas of the supports are beneficial to the dispersion of the catalytically active species. In recent years, our group has developed hierarchical carbon-based nanocages by in situ $\mathrm{MgO}$ template method. The mesostructured nanocages feature the high specific surface area, coexisting micro-meso-macropore structure, rich defects, easy doping, etc., which demonstrated excellent electrochemical performance in energy conversion and storage. Herein, taking advantage of the anchoring functions of nitrogen heteroatoms and high specific surface area of nitrogen-doped carbon nanocage (hNCNC), $10 \mathrm{wt} \% \mathrm{Ru} / \mathrm{hNCNC}$ catalyst was conveniently prepared by microwave-assisted ethylene glycol reduction. The Ru nanoparticles of $c a .2 .4 \mathrm{~nm}$ are highly dispersed on the outer surface of hNCNC. As the catalyst for the selective hydrogenation of acetophenone to 1-phenylethanol, $\mathrm{Ru} / \mathrm{hNCNC}$ exhibits excellent catalytic activity, selectivity and recyclability in mild conditions of $50.0{ }^{\circ} \mathrm{C}$
\end{abstract}

*E-mail: wqchem@nju.edu.cn; wangxzh@nju.edu.cn; Tel. \& Fax: +86-25-89693696; †前两位作者的贡献相同

Received March 31, 2017; published May 25, 2017

Project supported by the National Basic Research Program of China (973 Program, No. 2013CB932902), the National Natural Science Foundation of China (Nos. 21473089, 51232003, 21373108, 51571110, 21573107), Changzhou Technology Support Program (No. CE20130032), and the Priority Academic Program Development of Jiangsu Higher Education Institutions.

受国家重大科学研究计划(纳米专项, No. 2013CB932902), 国家自然科学基金(Nos. 21473089, 51232003, 21373108, 51571110, 21573107), 常州市科技 计划(No. CE20130032)和江苏高校优势学科建设工程项目资助. 
and 2.0 $\mathrm{MPa} \mathrm{H}_{2}$. Specifically, after $2.0 \mathrm{~h}$ of reaction, the conversion of acetophenone is up to $96.2 \%$, obviously higher than that of $\mathrm{Ru} /$ carbon nanocages $(\mathrm{Ru} / \mathrm{hCNC}, 80 \%)$ and $\mathrm{Ru} / \mathrm{AC}(0.7 \%)$, and the selectivity of 1-phenylethanol is $95.8 \%$. More importantly, after recycle use for 6 times, the conversion of acetophenone only slightly drops from $96.2 \%$ to $94.0 \%$ for $\mathrm{Ru} / \mathrm{hNCNC}$, while obviously decreases from $80.0 \%$ to $63.0 \%$ for $\mathrm{Ru} / \mathrm{hCNC}$. Such excellent catalytic performance of $\mathrm{Ru} / \mathrm{hNCNC}$ could be ascribed to the synergism of (i) the high dispersion of Ru nanoparticles owing to the high specific surface area and nitrogen doping of hNCNC, (ii) the regulated electron structure of Ru catalyst owing to nitrogen incorporation, iii) the facilitated mass transportation by unique hierarchical pore structures of hNCNC support.

Keywords acetophenone; selective hydrogenation; 1-phenylethanol; nitrogen-doped carbon nanocages; ruthenium

\section{1 引言}

共轭羰基化合物的羰基选择性加氢反应被广泛用 于制备重要的药物和化学中间体 ${ }^{[1 ~ 3]}$. 共轭羰基化合物 的加氢方式主要有两种: 一是采用氢化物(如 $\mathrm{LiAlH}_{4}$ 、 $\mathrm{NaBH}_{4}$ ) 作加氢试剂 ${ }^{[4]}$, 其加氢能力强, 但目标产物的选 择性不理想(源于共轭双键和羰基同时加氢)、氢化试剂 易燃易爆; 二是采用氢气作加氢试剂的羰基选择性催化 加氢或酮氢转移反应 ${ }^{[1,5 \sim 9]}$, 包括均相催化和非均相催 化过程. 以贵金属 $\left(\mathrm{Ru}^{[5 \sim 8]}\right.$ 和 $\mathrm{Pd}^{[1]}$ 等)有机配合物作催化 剂的均相过程具有催化活性好、目标产物选择性高等优 点, 但存在金属残留及贵金属催化剂难以回收等不足. 使用负载型非均相催化剂可以有效解决这些问题, 常用

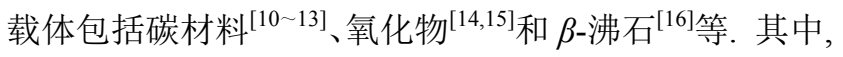
碳材料具有形态结构丰富、稳定性高、比表面积和孔结 构可调、易于掺杂等特点, 是构建高性能催化剂的优良 载体. 研究表明, 载体的高比表面积有利于催化活性物 种的分散, 而在石墨碳层中引入杂原子能进一步促进其 分散、并调节金属与载体之间的相互作用, 从而调变其 催化性能 ${ }^{[17 ~ 19]}$. 近年来, 本课题组发展原位 $\mathrm{MgO}$ 模板 法制得了具有比表面积大、微孔一介孔一大孔共存、易于 掺杂等特点的分级结构碳基纳米笼新材料, 在能源转化 和存储等方面表现出优异性能 ${ }^{[20-22]}$.

本文以分级结构氮掺杂碳纳米笼 $(\mathrm{hNCNC})$ 为载体, 利用其高比表面积和掺杂氮原子的针定作用, 通过微波 辅助乙二醇还原法构建了 $\mathrm{Ru}$ 纳米粒子高度分散的 $\mathrm{Ru} / \mathrm{hNCNC}$ 催化剂, 用于催化苯乙酮选择性加氢制 1-苯 乙醇的模型反应, 在温和条件下展现出高的苯乙酮转化 率(可接近 100\%)、优良的 1-苯乙醇选择性(超过 95\%) 及循环稳定性, 显著优于 $\mathrm{Ru} /$ 碳纳米笼 $(\mathrm{hCNC})$ 和 $\mathrm{Ru} / \mathrm{AC}$ 催化剂. 其优异催化性能主要源于: hNCNC大的比表面 积和掺杂氮原子有利于 $\mathrm{Ru}$ 纳米粒子的分散和针定, 载 体中微孔-介孔-大孔共存的多级孔结构有利于传质, 掺 杂氮原子有效调变了 $\mathrm{Ru}$ 纳米粒子的电子结构.

\section{2 结果与讨论}

采用微波辅助乙二醇还原法制得了 $\mathrm{Ru} / \mathrm{hNCNC}$ 、 $\mathrm{Ru} / \mathrm{hCNC}$ 和 $\mathrm{Ru} / \mathrm{AC}$ 催化剂, 其中 $\mathrm{Ru}$ 的负载量均为 10 $\mathrm{wt} \%$. 图 1 是 $\mathrm{hNCNC}$ 载体和上述催化剂的形貌和结构 表征结果. 扫描电镜(SEM)结果表明, hNCNC 和 hCNC
均是由空心纳米笼相互连成纳米片、再由纳米片组装而 成的分级介观结构; 高分辨透射电镜(HRTEM)观察表 明其具有良好石墨化程度(图 1a), 与我们之前的报道类 似 ${ }^{[21,22]}$; 而 $\mathrm{AC}$ 为纳米碳的随机密堆结构(图 1e). 其比表 面积依次为 $1150 、 1230$ 和 $1730 \mathrm{~m}^{2} \cdot \mathrm{g}^{-1}$, 如此高的比表 面积应有利于 $\mathrm{Ru}$ 纳米粒子的分散. $\mathrm{hNCNC}$ 和 $\mathrm{hCNC}$ 的 氮气吸脱附等温线呈 IV 型特征, 表明其具有微孔、介孔 和大孔共存的特征, 富含 $2 \sim 20 \mathrm{~nm}$ 的介孔; $\mathrm{AC}$ 的氮气 吸脱附等温线呈 I 型特征, 主要是 $<5 \mathrm{~nm}$ 的微孔和介 孔(图 1b). 相比较而言, hNCNC 和 $\mathrm{hCNC}$ 的多尺度孔结 构便于反应物种的输运, 对催化反应有利. 图 $1 \mathrm{c} \sim 1 \mathrm{e}$ 是 $\mathrm{Ru} / \mathrm{hNCNC} 、 \mathrm{Ru} / \mathrm{hCNC}$ 和 $\mathrm{Ru} / \mathrm{AC}$ 催化剂的 TEM 照片, 可 以看到, $\mathrm{Ru}$ 纳米粒子高度均匀分散在各载体表面, 其尺 寸依次为 $2.4 \pm 0.5 、 4.9 \pm 1.0$ 和 $1.3 \pm 0.3 \mathrm{~nm}$; HRTEM 照 片显示纳米粒子的晶面间距为 $0.207 \mathrm{~nm}$ (图 1c), 对应于 六方相金属 Ru 的(101)晶面(JCPDS No. 88-1734), 表明 纳米粒子为金属态 $\mathrm{Ru}(0)$. XRD 图中未见金属 $\mathrm{Ru}$ 及其化 合物的衍射峰(图 1f), 可能是由于 $\mathrm{Ru}$ 纳米粒子的晶粒尺 寸小(图 1c $\sim 1 \mathrm{e}$ 的粒子尺寸统计图), 其衍射信号弥散在 背景中.

载体和 $\mathrm{Ru}$ 基催化剂的 XPS 结果见图 2. 图 2a 的 XPS 全谱表明, $\mathrm{MgO}$ 模板被完全去除; hNCNC 的氮含量 为 12.4 at. $\%$; Ru/hNCNC 中 $\mathrm{Ru}$ 含量为 $10.5 \mathrm{wt} . \%$, 与 $\mathrm{Ru}$ 的投料量一致, 说明 $\mathrm{Ru}(\mathrm{III})$ 离子几乎完全被还原并沉积 到载体表面. hNCNC 的 N 1s XPS 谱可去卷积为吡啶氮 $(398.3 \mathrm{eV})$ 、吡咯氮 $(400.0 \mathrm{eV})$ 、石墨氮 $(401.0 \mathrm{eV})$ 和 N氧化物(403.8 eV)四种形态(图 2b) ${ }^{[21,22]} ; \mathrm{N}$ 的引入有利于 $\mathrm{Ru}$ 纳米粒子的固载和分散 ${ }^{[17,18,23,24]}$, 相比于 $\mathrm{hCNC}$ 载体, $\mathrm{hNCNC}$ 负载的 $\mathrm{Ru}$ 纳米粒子具有更小的尺寸(图 1c $1 \mathrm{~d}$ ); 负载 Ru 之后, N1s 向高结合能方向偏移约 $0.2 \mathrm{eV}$, 说明有电子从载体向 $\mathrm{Ru}$ 纳米粒子转移. $\mathrm{Ru} / \mathrm{hCNC}$ 的 $\mathrm{Ru}$ $3 p_{3 / 2}$ 谱可去卷积为 $R u(0)(463.1 \mathrm{eV})$ 和 $\mathrm{Ru}(\mathrm{IV})(466.1 \mathrm{eV})$ 物种, 其中金属态 $\mathrm{Ru}(0)$ 占 $80.7 \%$, 与 HRTEM 揭示的纳 米粒子为金属态 $\mathrm{Ru}(0)$ 的结果是一致的(图 1c); 相比之 下, $\mathrm{hNCNC}$ 负载的 $\mathrm{Ru}$ 向低结合能方向偏移了约 $0.3 \mathrm{eV}$, 说明 $\mathrm{Ru}$ 纳米粒子从载体获得了电子. 该结果表明, $\mathrm{Ru}$ 和 $\mathrm{hNCNC}$ 之间存在比 $\mathrm{Ru}$ 和 $\mathrm{hCNC}$ 更强的相互作 用 ${ }^{[23,24]}$. 图 $2 \mathrm{~d}$ 的 Raman 光谱表明, 载体 $\mathrm{hNCNC}$ 和 $\mathrm{hCNC}$ 均存在代表缺陷的 D 带(约 $1350 \mathrm{~cm}^{-1}$ )和代表石墨 化程度的 $\mathrm{G}$ 带(约 $\left.1580 \mathrm{~cm}^{-1}\right)^{[13]}$, 且 $\mathrm{N}$ 掺杂后 $\mathrm{hCNC}$ 的 

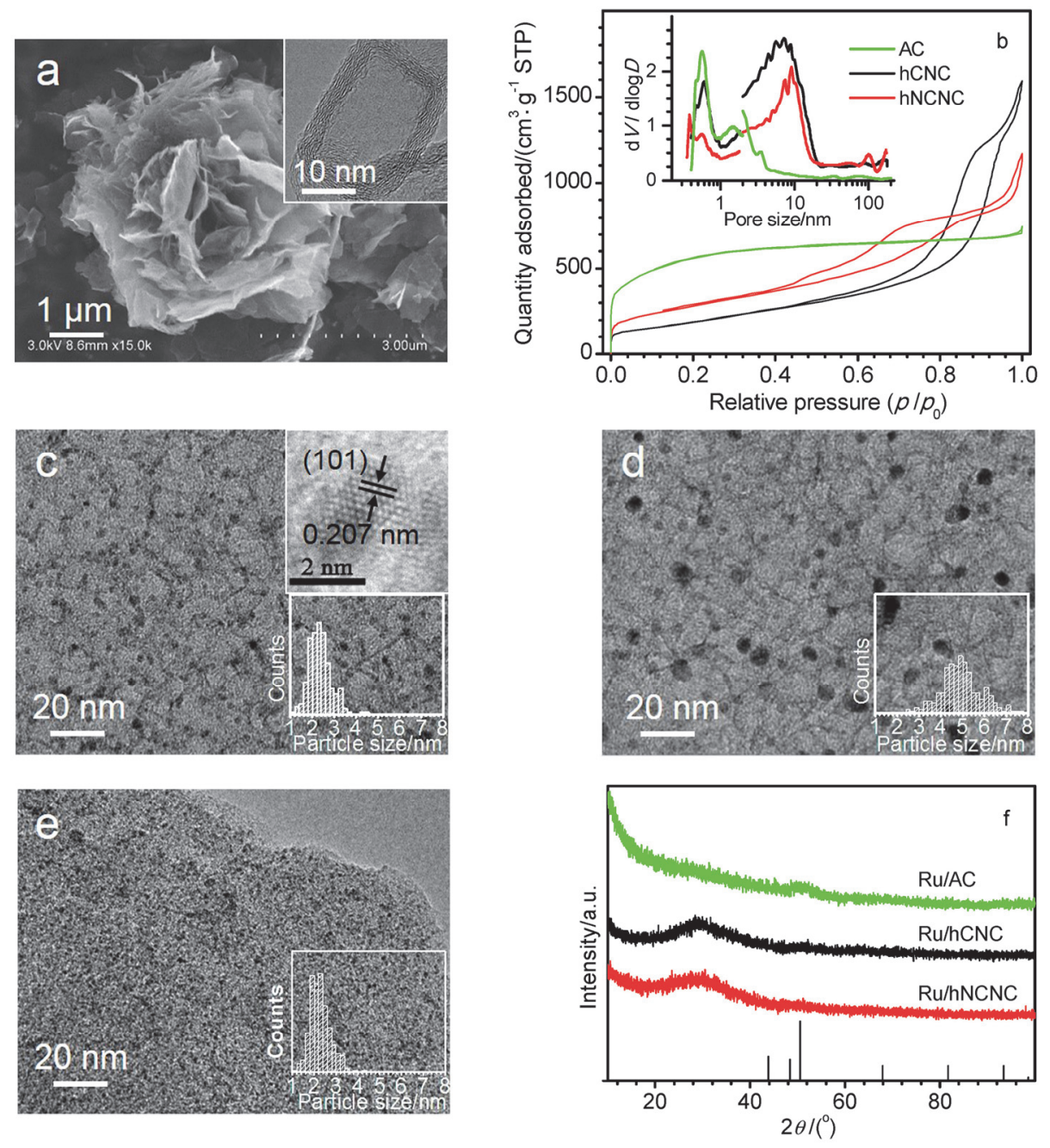

图 1 载体和 $\mathrm{Ru}$ 基催化剂的形貌和结构表征

Figure 1 Morphologies and structures of supports and Ru-based catalysts

(a) SEM image of hNCNC. Inset is HRTEM image. (b) $\mathrm{N}_{2}$ adsorption/desorption isotherms of hNCNC, hCNC and AC. The inset is the corresponding plot of pore size distributions. ( $\sim$ e) TEM images of Ru/hNCNC, Ru/hCNC and Ru/AC. The insets are HRTEM images and histograms of particle size distribution from $c a$. 300 Ru nanoparticles. (f) Corresponding XRD patterns

$D$ 带和 $G$ 带的半峰宽均明显变大、相应的峰面积比 $\left(I_{\mathrm{D}} / I_{\mathrm{G}}\right)$ 亦从 2.2 增加到 3.0. 这表明二者均有较好的石墨 化程度和较多的缺陷, 这与 HRTEM 的观察结果是一致 的(图 1a), N 掺杂能在 hCNC 中引入更多缺陷.

苯乙酮的加氢产物包括 1-苯乙醇 $(\mathbf{1}$, 目标产物)、1环己基乙酮(2)和 1-环己基乙醇(3). 在不同催化剂作用 下, 苯乙酮的转化率(催化活性)、各产物的选择性和裸

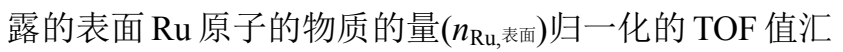
列于表 1. 在 $2.0 \mathrm{MPa} \mathrm{H}_{2} 、 50.0{ }^{\circ} \mathrm{C}$ 的温和条件下, 反应 $2.0 \mathrm{~h}$ 后, Ru/hNCNC 催化剂的苯乙酮转化率为 $96.2 \%$, 高于 $\mathrm{Ru} / \mathrm{hCNC}(80.0 \%)$ 和 $\mathrm{Ru} / \mathrm{AC}(0.7 \%)$, 目标产物 1-苯 乙醇的选择性均超过 $95.0 \%$. 根据 $\mathrm{Ru}$ 纳米粒子平均粒 径(图 $1 \mathrm{c} \sim 1 \mathrm{e}$ )、催化剂用量和负载量, 可以估算出 $n_{\mathrm{Ru}, \text { 表面 }}$ 依次为 $1.36 \times 10^{-6} 、 6.68 \times 10^{-7}$ 和 $2.52 \times 10^{-6} \mathrm{~mol}$, 再假设裸露的 $\mathrm{Ru}$ 原子均是活性位点, 可以估算出它们
在反应 $1 \mathrm{~h}$ 内的平均 TOF 值分别为: 504、453 和 1.2

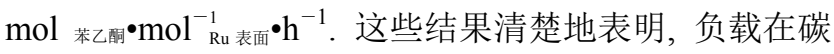
基纳米笼( $\mathrm{hNCNC}$ 和 $\mathrm{hCNC}$ )上的 $\mathrm{Ru}$ 催化剂的活性远超 过负载于 $\mathrm{AC}$ 的 $\mathrm{Ru}$ 催化剂, 这可归因于 $\mathrm{hNCNC}$ 和 $\mathrm{hCNC}$ 载体的微孔一介孔-大孔共存的介观结构有利于传 质; $\mathrm{AC}$ 微孔和介孔发达, 而缺乏有利于传质的介孔和大 孔(图 1a, 1b), 且部分 $\mathrm{Ru}$ 纳米粒子被包埋在 $\mathrm{AC}$ 的这些 $<5 \mathrm{~nm}$ 的微孔和介孔中, 活性位暴露不充分, 导致催化 活性难以发挥 ${ }^{[25]}$. 有意思的是, $\mathrm{Ru} / \mathrm{hNCNC}$ 的苯乙酮转 化率和平均 TOF 值 $(1 \mathrm{~h})$ 均高于 $\mathrm{Ru} / \mathrm{hCNC}$ (表 1), 这是因 为氮掺杂原子的错定作用使 $\mathrm{Ru}$ 纳米粒子高度分散并减 小其晶粒尺寸(图 1c, 1d), 且调节了 $\mathrm{Ru}$ 催化剂的电子结 构(图 2b, 2c). 此外, 苯乙酮与催化剂中 $\mathrm{Ru}$ 的质量比高 达约 450, 表明 Ru/hNCNC 是催化苯乙酮加氢到 1-苯乙 醇的高效催化剂. 

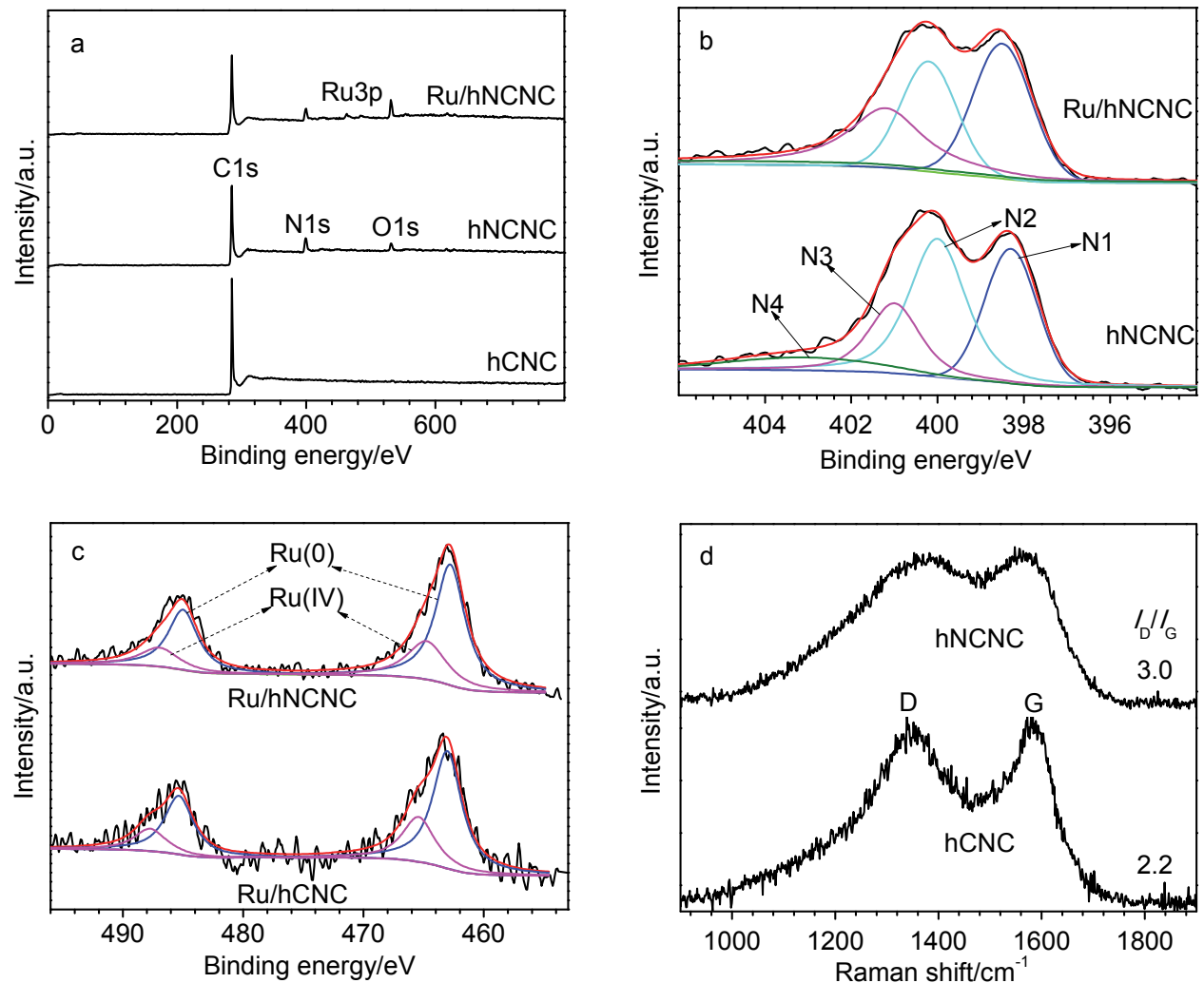

图 2 载体和 $\mathrm{Ru}$ 基催化剂的 XPS 谱

Figure 2 XPS spectra of supports and Ru-based catalysts

(a) XPS survey spectrum of Ru/hNCNC, hNCNC and hCNC. (b) The deconvolution plot of N1s of hNCNC and Ru/hNCNC. N1, N2, N3, and N4 denote pyridinic, pyrrolic, graphitic nitrogen, and pyridinic N-oxide species, respectively. (c) The deconvolution plot of Ru 3p XPS spectrum of Ru/hCNC and Ru/hNCNC. (d) Raman scatter of hNCNC and hCNC

表 1 催化苯乙酮选择性加氢性能 ${ }^{a}$

Table 1 Selectively catalytic hydrogenation of acetophenone

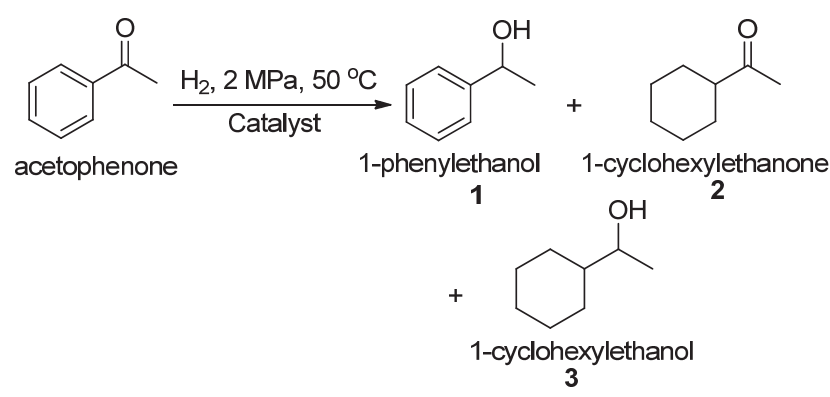

\begin{tabular}{|c|c|c|c|c|c|}
\hline \multirow{2}{*}{ Catalyst } & \multirow{2}{*}{$\begin{array}{c}\text { Conversion/ } \\
\% \\
\end{array}$} & \multicolumn{3}{|c|}{ Selectivity $/ \%$} & \multirow{2}{*}{ 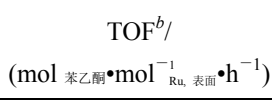 } \\
\hline & & 1 & 2 & 3 & \\
\hline $\mathrm{hNCNC}$ & 0 & 0 & 0 & 0 & 0 \\
\hline $\mathrm{Ru} / \mathrm{hNCNC}$ & 96.2 & 95.8 & 1.0 & 3.2 & 504 \\
\hline $\mathrm{Ru} / \mathrm{hCNC}$ & 80.0 & 95.0 & 0.7 & 4.3 & 453 \\
\hline $\mathrm{Ru} / \mathrm{AC}$ & 0.7 & 100.0 & 0 & 0 & 1.2 \\
\hline
\end{tabular}

${ }^{a}$ Reaction conditions: $0.10 \mathrm{~mL}$ acetophenone $(0.85 \mathrm{mmol}), 5.0 \mathrm{~mL}$ isopropanol, $2.0 \mathrm{mg}$ catalysts, $2.0 \mathrm{MPa} \mathrm{H}_{2}, 50.0{ }^{\circ} \mathrm{C}, 2.0 \mathrm{~h} .{ }^{b} \mathrm{TOF}$ is defined as the amount of substance of converted acetophenone divided by both reaction time $(1 \mathrm{~h})$ and the amount of substance of the exposed surface Ru atoms $\left(n_{\mathrm{Ru}}\right.$, surface). The $n_{\mathrm{Ru} \text {, surface }}$ value is estimated by the average diameter of Ru nanoparticles, density and radii of $\mathrm{Ru}$.

为了进一步揭示氮掺杂对碳纳米笼负载 $\mathrm{Ru}$ 催化剂
的促进作用, 对比研究了 $\mathrm{Ru} / \mathrm{hNCNC}$ 和 $\mathrm{Ru} / \mathrm{hCNC}$ 催化 苯乙酮加氢反应的循环稳定性, 结果见图 3. 循环使用 6 次后, Ru/hNCNC 的苯乙酮转化率仅从 $96.2 \%$ 略微下降 到 $94.0 \%$ ，而 $\mathrm{Ru} / \mathrm{hCNC}$ 则从 $80.0 \%$ 显著下降到 $63.0 \%$ (图 $3 \mathrm{a})$. 可见, 氮的掺杂显著提高了 $\mathrm{Ru}$ 催化剂的催化活性 及其稳定性. 用 TEM 表征了经 6 次循环使用后的催化 剂, 发现 $\mathrm{Ru} / \mathrm{hNCNC}$ 催化剂中 $\mathrm{Ru}$ 纳米粒子保持均匀分 散, 其尺寸为 $2.1 \pm 0.5 \mathrm{~nm}$ (图 3b), 与新鲜催化剂的 2.4 $\pm 0.5 \mathrm{~nm}$ 大致相当(图 1c); Ru/hCNC 催化剂中仅见到尺 寸 $<1 \mathrm{~nm}$ 的 $\mathrm{Ru}$ 纳米粒子(图 3c), 远小于新鲜催化剂中 的尺寸 $(4.9 \pm 1.0 \mathrm{~nm})$ (图 1d), 说明大部分 $\mathrm{Ru}$ 纳米粒子经 过 6 次循环后已从 $\mathrm{hCNC}$ 表面脱落, 导致催化活性大幅 下降. 这一结果表明, 载体中掺杂氮原子与 $\mathrm{Ru}$ 纳米粒 子之间的强相互作用使其稳定性显著提升(图 2).

本文还系统考察了 $\mathrm{H}_{2}$ 压强、反应温度和反应时间 对 $\mathrm{Ru} / \mathrm{hNCNC}$ 的活性和产物选择性的影响, 结果示于图 4. 在 $50.0{ }^{\circ} \mathrm{C} 、 2.0 \mathrm{~h}$ 反应条件下, 当 $\mathrm{H}_{2}$ 压强为 $1.0 、 2.0$ 、 3.0 和 $4.0 \mathrm{MPa}$ 时，苯乙酮转化率分别为 $81.0 \% 、 96.2 \%$ 、 $99.5 \%$ 和 $99.7 \%$ ，1-苯乙醇的选择性为 $96.2 \% 、 95.8 \%$ 、 $94.0 \%$ 和 $93.0 \%$ (图 4a); 可见, 当 $\mathrm{H}_{2}$ 压强达到 $2.0 \mathrm{MPa}$ 后, 苯乙酮转化率相近, 但 1-苯乙醇的选择性略有下降, 因此优化的 $\mathrm{H}_{2}$ 压强为 $2.0 \mathrm{MPa}$. 在此 $\mathrm{H}_{2}$ 压强下, 反应温 

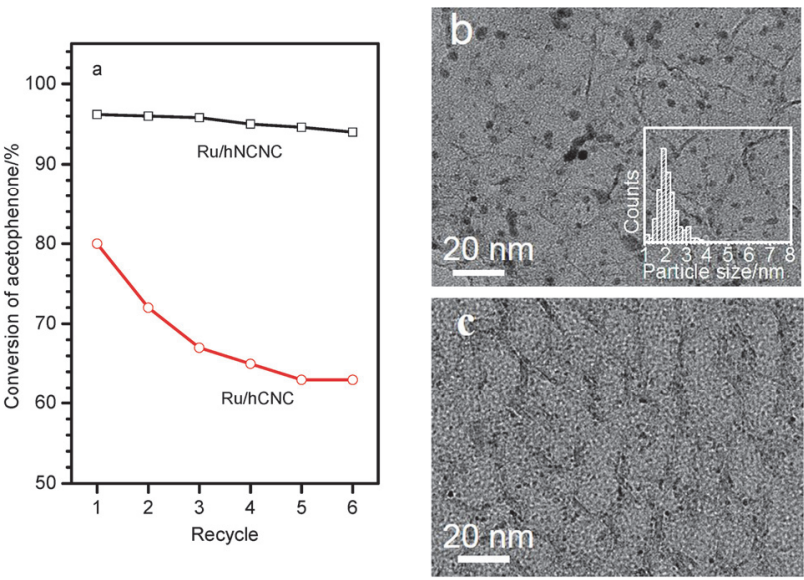

图 $3 \mathrm{Ru} / \mathrm{hNCNC}$ 和 $\mathrm{Ru} / \mathrm{hCNC}$ 的可循环使用性能及其 TEM 表征

Figure 3 Recyclability and TEM characterizations of Ru/hNCNC and $\mathrm{Ru} / \mathrm{hCNC}$ catalysts

(a) Plot of conversion of acetophenone versus cycle number. (b, c) TEM images of post-reacted $\mathrm{Ru} / \mathrm{hNCNC}$ and $\mathrm{Ru} / \mathrm{hCNC}$ catalysts. The insert is the histogram of particle size distribution from $c a .300$ Ru nanoparticles. Reaction conditions: $0.10 \mathrm{~mL}$ acetophenone, $5.0 \mathrm{~mL}$ isopropanol, $2.0 \mathrm{mg}$ catalysts, 2.0 $\mathrm{MPa} \mathrm{H}_{2}, 50{ }^{\circ} \mathrm{C}$, and $2.0 \mathrm{~h}$

度和反应时间对苯乙酮转化率和 1-苯乙醇选择性的影 响见图 4b,4c. 可以看到, 随反应温度增加, 相同反应 时间内苯乙酮转化率上升, 1-苯乙醇选择性下降, 但仍 然保持超过 $89 \%$ 的高选择性; 在相同的反应温度, 随反
应时间延长, 苯乙酮转化率上升而 1-苯乙醇选择性略有 下降. 我们注意到反应温度为 $50.0{ }^{\circ} \mathrm{C}$ 、反应时间为 $2.0 \mathrm{~h}$ 时, 苯乙酮转化率已经达到 $96.2 \%, 1$-苯乙醇选择性可达 $95.8 \%$. 因此, 优化反应条件为: $2.0 \mathrm{MPa} \mathrm{H}_{2} 、 50.0{ }^{\circ} \mathrm{C}$ 和 $2.0 \mathrm{~h}$. 该反应条件相对温和, 优于文献报道 ${ }^{[26]}$. 为揭示 反应温度对 $\mathrm{Ru} / \mathrm{hNCNC}$ 和 $\mathrm{Ru} / \mathrm{hCNC}$ 催化性能的影响规 律, 在 $2.0 \mathrm{MPa} \mathrm{H}_{2}$ 和 $2.0 \mathrm{~h}$ 的反应条件下的催化性能结 果见图 4d. 可以看到, 二者的 1-苯乙醇选择性均超过 $90 \%$, 随反应温度的升高, 苯乙酮转化率均显著升高, 1苯乙醇选择性均略有下降; 除 $30{ }^{\circ} \mathrm{C}$ 之外, $\mathrm{Ru} / \mathrm{hNCNC}$ 的 苯乙酮转化率均高于 $\mathrm{Ru} / \mathrm{hCNC}$. 这一结果进一步表明, 在 $\mathrm{hCNC}$ 中引入掺杂氮原子, 能显著提升其负载 $\mathrm{Ru}$ 纳 米粒子后催化剂的催化性能.

上述结果表明, 以 $\mathrm{hNCNC}$ 为载体, 掺杂氮原子和 大比表面积有利于 $\mathrm{Ru}$ 纳米粒子的高度分散和针定, 载 体的多级孔结构有利于液相传质，掺杂氮原子还有效调 变了 $\mathrm{Ru}$ 纳米粒子的电子结构, 这些特性使 $\mathrm{Ru} / \mathrm{hNCNC}$ 在温和条件下展现出苯乙酮催化加氢制 1-苯乙醇的优 异催化性能, 显著优于未掺杂的 $\mathrm{hCNC}$ 和 $\mathrm{AC}$ 载体.

\section{3 结论}

利用掺杂氮原子的针定作用和 $\mathrm{hNCNC}$ 的高比表 面积可方便地将约 $2.4 \mathrm{~nm}$ 的 $\mathrm{Ru}$ 纳米粒子均匀地固载在
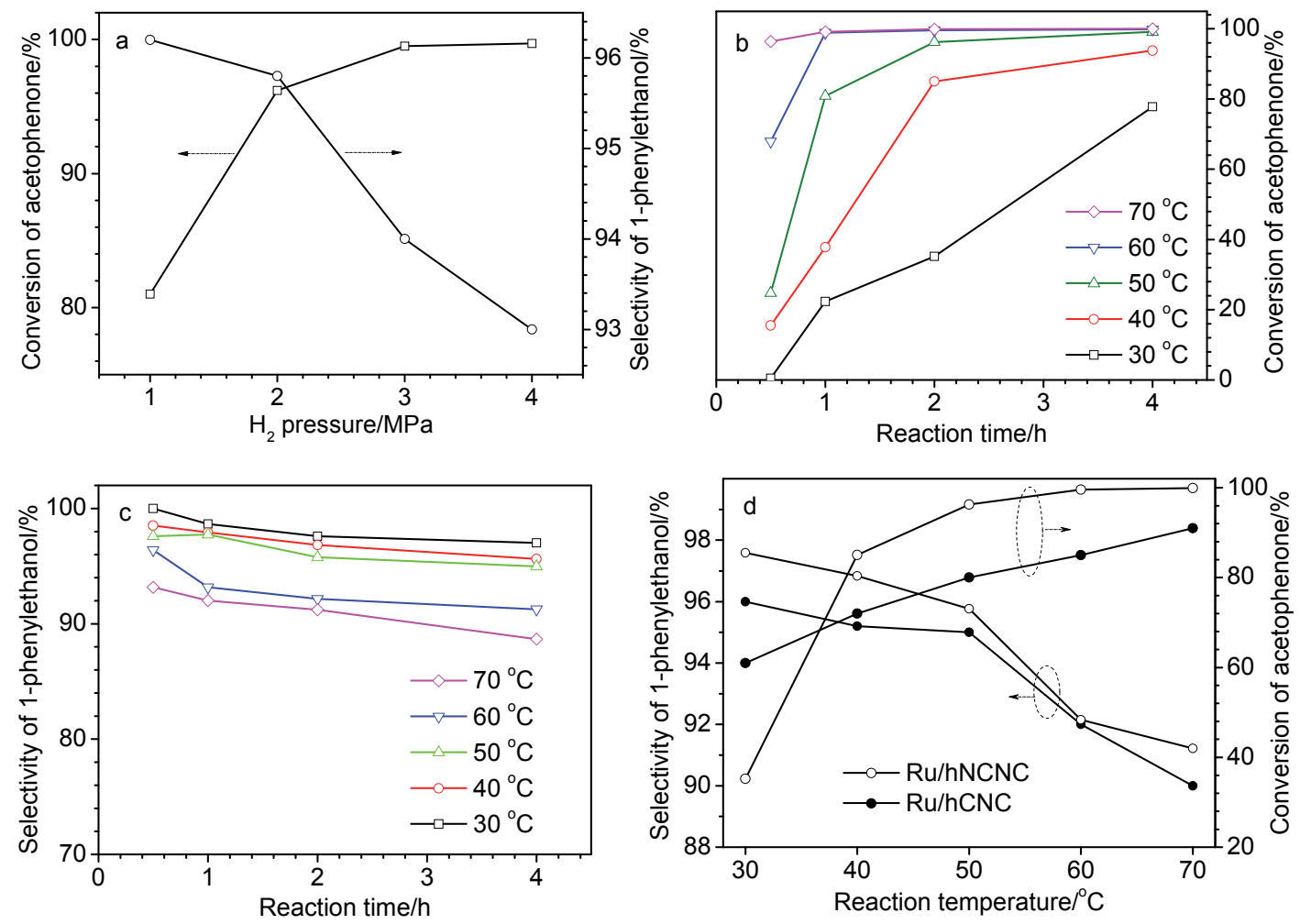

图 $4 \mathrm{H}_{2}$ 压强(a)、温度(b)、时间(c)和载体(d)对苯乙酮转化率和 1-苯乙醇选择性的影响

Figure 4 Effect of hydrogen pressure (a), reaction temperature (b), time (c), and support (d) on conversion of acetophenone and selectivity of 1-phenylethanol

Reaction conditions: $0.10 \mathrm{~mL}$ acetophenone, $5.0 \mathrm{~mL}$ isopropanol, $2.0 \mathrm{mg}$ catalyst. $\mathrm{Ru} / \mathrm{hNCNC}$ in $(\mathrm{a} \sim \mathrm{c}) .2 .0 \mathrm{~h}$ and $50.0{ }^{\circ} \mathrm{C}$ in $(\mathrm{a}) .2 .0 \mathrm{MPa} \mathrm{H}_{2}$ in $(\mathrm{b}, \mathrm{c}) . \mathrm{Ru} / \mathrm{hNCNC}$ or $\mathrm{Ru} / \mathrm{hNCNC}, 2.0 \mathrm{~h}$, and $2.0 \mathrm{MPa} \mathrm{H}_{2}$ in (d) 
hNCNC 表面. 用于苯乙酮选择性加氢制 1-苯乙醇的反 应, $\mathrm{Ru} / \mathrm{hNCNC}$ 表现出优异的催化性能, 在 $2.0 \mathrm{MPa} \mathrm{H}_{2}$ 、 $50.0{ }^{\circ} \mathrm{C}$ 的温和条件下, $2.0 \mathrm{~h}$ 的苯乙酮转化率和 1 -苯乙 醇选择性分别达到 $96.2 \%$ 和 $95.8 \%$, 远优于未掺杂 $\mathrm{hCNC}$ 和 $\mathrm{AC}$ 负载的 $\mathrm{Ru}$ 催化剂; 循环使用 6 次后, 其苯 乙酮转化率仅轻微下降, 而 $\mathrm{Ru} / \mathrm{hCNC}$ 的苯乙酮转化率 从 $80.0 \%$ 下降到 $63.0 \% \mathrm{Ru} / \mathrm{hNCNC}$ 催化剂的优异催化性 能源自以下因素的共同作用: hNCNC 的大比表面积和 掺杂氮原子有利于 $\mathrm{Ru}$ 纳米粒子的分散和针定, 载体中 微孔一介孔-大孔共存的多级孔结构有利于传质, 掺杂氮 原子有效调变了 $\mathrm{Ru}$ 纳米粒子的电子结构.

\section{4 实验部分}

\section{1 催化剂制备}

分级结构碳基纳米笼是采用我们课题组发展的原 位 $\mathrm{MgO}$ 模板法制备的 ${ }^{[21,22]}$. 具体来说, $4.0 \mathrm{~g}$ 具有分级结 构的碱式碳酸镁 $\left(4 \mathrm{MgCO}_{3} \cdot \mathrm{Mg}(\mathrm{OH})_{2} \cdot 5 \mathrm{H}_{2} \mathrm{O}\right)$ 放入坚直的 石英反应管内, 在 $100 \mathrm{sccm}$ 的氩气气氛下, 以 $10{ }^{\circ} \mathrm{C} \cdot$ $\min ^{-1}$ 的速率升至 $800{ }^{\circ} \mathrm{C}$, 用恒流洜以 $60 \mu \mathrm{L} \cdot \mathrm{min}^{-1}$ 的流 量注入吡啶前驱体 $5.0 \mathrm{~min}$, 保温 $1.0 \mathrm{~h}$, 自然冷却到室 温. 用 $6 \mathrm{~mol} \cdot \mathrm{L}^{-1} \mathrm{HCl}$ 溶液去除 $\mathrm{MgO}$ 模板, 搅拌 $48.0 \mathrm{~h}$ 、 过滤、去离子水反复洗涤、 $80{ }^{\circ} \mathrm{C}$ 干燥 $24.0 \mathrm{~h}$, 所得产物 标记为 hNCNC. 对照组的 hCNC 采用相同的流程制备, 仅仅是把吡啶换成了苯.

采用微波辅助乙二醇还原法, 制备 $\mathrm{Ru}$ 负载量为 10 $\mathrm{wt} \%$ 的 $\mathrm{Ru} / \mathrm{hNCNC} 、 \mathrm{Ru} / \mathrm{hCNC}$ 和 $\mathrm{Ru} / \mathrm{AC}$ 催化剂. 具体流 程: 在 $50 \mathrm{~mL}$ 乙二醇中加入 $100 \mathrm{mg}$ 载体, 超声 $30 \mathrm{~min}$ 使其分散均匀; 将含有 $8.4 \mathrm{mg} \mathrm{Ru}$ 的 $\mathrm{RuCl}_{3}$ 乙二醇溶液 逐滴加入到悬浮液中, 磁力搅拌 $4 \mathrm{~h}$; 逐滴加入适量 0.2 $\mathrm{mol} \cdot \mathrm{L}^{-1} \mathrm{NaOH} /$ 乙二醇溶液, 直到 $\mathrm{pH}=8$, 磁力搅拌 30 $\mathrm{min}$; 将悬浮液放入 $750 \mathrm{~W}$ 微波炉(Galanz)中辐照 $120 \mathrm{~s}$, 过滤、去离子水和乙醇洗涤数次、 $100{ }^{\circ} \mathrm{C}$ 真空干燥 $12 \mathrm{~h}$.

\section{2 形貌、结构和组成表征}

载体和催化剂的形貌、结构和成分表征是在高分辨 透射电子显微镜(HRTEM, JEM-2100F, $200 \mathrm{kV}$ )、X 射线 衍射(XRD, Bruker, D8 Advance A25, Co 靶, $K_{\alpha 1}=$ $1.78897 \AA, 0.02 \mathrm{~mm}$ 厚度 $\mathrm{Fe}$ 滤光片)和 $\mathrm{X}$ 射线光电子能 谱(XPS, PHI 5000 VersaProbe). 氮气吸/脱附等温线是在 Thermo Fisher Scientific Surfer Gas Adsorption Porosimeter 上测试的 $(77 \mathrm{~K})$, 样品在 $10^{-3}$ torr、 $300.0{ }^{\circ} \mathrm{C}$ 条件下 预处理 $6.0 \mathrm{~h}$; 比表面积和孔分布分别由 Brunauer-Emmett-Teller (BET, $p / p_{0}: 0.05 \sim 0.35$ )、Horvath-Kawazoe $(\mathrm{HK},<2 \mathrm{~nm}$ 微孔)和 Barrett-Joyner-Halenda (BJH, >2 $\mathrm{nm}$ 介孔)方法处理吸附支数据得到的.

\section{3 催化性能表征}

将 $0.10 \mathrm{~mL}(0.85 \mathrm{mmol})$ 苯乙酮和 $2.0 \mathrm{mg}$ 催化剂加
入 $100 \mathrm{~mL}$ 高压反应釜中, 加 $5.0 \mathrm{~mL}$ 异丙醇溶剂, 充入 $\mathrm{H}_{2}$ 至指定压强 $(1.0 \sim 4.0 \mathrm{MPa})$, 升温至指定温度(30.0 $\left.70.0{ }^{\circ} \mathrm{C}\right)$, 磁力摚拌下, 反应一段时间 $(0.5 \sim 4.0 \mathrm{~h})$. 反应 结束后, 用 $0.22 \mu \mathrm{m}$ 孔径的聚四氟乙烯(PTFE)滤膜过滤. 回收的催化剂直接做可重复利用性实验, 反应条件为: $2.0 \mathrm{MPa} \mathrm{H}_{2} 、 50.0{ }^{\circ} \mathrm{C}$ 和 $2.0 \mathrm{~h}$, 其他条件不变. 取 $0.50 \mathrm{~mL}$ 滤液, 加入等体积乙醇、 $100 \mu \mathrm{L}$ 均三甲苯内标物, 混合 均匀, 在气相色谱一质谱联用(GCMS-QP2010, 色谱柱: DB-5MS, 载气: He)、气相色谱(GC-7900, 色谱柱: HP-5, 载气: $\mathrm{N}_{2}$ )做定性和定量分析.

\section{References}

[1] Chen, Q. A.; Ye, Z. S.; Duan, Y.; Zhou, Y. G. Chem. Soc. Rev. 2013, $42,497$.

[2] Mäki-Arvela, P.; Hájek, J.; Salmi, T.; Murzin, D. Y. Appl. Catal., A $\mathbf{2 0 0 5}, 292,1$.

[3] Gallezot, P.; Richard, D. Catal. Rev. 1998, 40, 81.

[4] Brown, H. C.; Ramachandran, P. V. In Reductions in Organic Synthesis, Ed.: American Chemical Society, Washington, DC, 1996, Chapter 1.

[5] Noyori, R.; Ohkuma, T. Angew. Chem., Int. Ed. 2001, 40, 40.

[6] Gao, A. L.; Ye, Q. S.; Yu, J.; Liu, W. P. Chin. J. Org. Chem. 2017, 37, 47. (高安丽, 叶青松, 余娟, 刘伟平, 有机化学, 2017, 37, 47.)

[7] Li, X. N.; Wang, L. H.; Zhou, H. Y.; Wang, J. X. Chin. J. Org. Chem. 2016, 36, 2175. (李小娜, 王丽华, 周宏勇, 王家喜, 有机化学, 2016, 36, 2175.)

[8] Dai, N.; Shang, R.; Fu, M. C.; Fu, Y. Chin. J. Chem. 2015, 33, 405

[9] Liang, M. T.; Xia, X. F.; Liu, X.; Li, H. X. Chin. J. Chem. 2015, 33, 578.

[10] Toebes, M. L.; Zhang, Y. H.; Hájek, J.; Nijhuis, T. A.; Bitter, J. H.; van Dillen, A. J.; Murzin, D. Y.; Koningsberger, D. C.; de Jong K. P. J. Catal. 2004, 226, 215.

[11] Bergault, I.; Fouilloux, P.; Joly-Vuillemin, C.; Delmas, H. J. Catal. 1998, 175, 328

[12] Zhao, B. H.; Chen, J. G.; Liu, X.; Liu, Z. W.; Hao, Z. P.; Xiao, J. L.; Liu, Z. T. Ind. Eng. Chem. 2012, 51, 11112.

[13] Gopiraman, M.; Babu, G. S.; Khatri, Z.; Kai, W.; Kim, Y. A.; Endo, M.; Karvembu, R.; Kim, I. S. J. Phys. Chem. C 2013, 117, 23582.

[14] Xiong, W.; Huang, Y. Y.; Chen, H.; Li, X. J. Acta Chim. Sinica 2005, 63，1927. (熊伟，黄艳轶，陈华，李贤均，化学学报， 2005，63， 1927.)

[15] Malathi, R.; Viswanath, R. P. Appl. Catal. A 2001, 208, 323.

[16] Kantam, M. L.; Rao, B. P. C.; Choudary, B. M.; Sreedhar, B. Adv. Synth. Catal. 2006, 348, 1970.

[17] Yue, B.; Ma, Y. W.; Tao, H. S.; Yu, L. S.; Jian, G. Q.; Wang, X. Z.; Wang, X. S.; Lu, Y. N.; Hu, Z. J. Mater. Chem. 2008, 18, 1747.

[18] Feng, H.; Ma, J.; Hu, Z. J. Mater. Chem. 2010, 20, 1702.

[19] Ning, X. M.; Li, Y. H.; Dong, B. Q.; Wang, H. J.; Yu, H.; Peng, F.; Yang, Y. H. J. Catal. 2017, 348, 100.

[20] Xie, K.; Qin, X. T.; Wang, X. Z.; Wang, Y. N.; Tao, H. S.; Wu, Q.; Yang, L. J.; Hu, Z. Adv. Mater. 2012, 24, 347.

[21] Chen, S.; Bi, J. Y.; Zhao, Y.; Yang, L. J.; Zhang, C.; Ma, Y. W.; Wu, Q.; Wang, X. Z.; Hu, Z. Adv. Mater. 2012, 24, 5593.

[22] Zhao, J.; Lai, H. W.; Lyu, Z. Y.; Jiang, Y. F.; Xie, K.; Wang, X. Z.; Wu, Q.; Yang, L. J.; Jin, Z.; Ma, Y. W.; Liu, J.; Hu, Z. Adv. Mater. $\mathbf{2 0 1 5}, 27,3541$

[23] Jiang, S. J.; Zhu, L.; Ma, Y. W.; Wang, X. Z.; Liu, J. G.; Zhu, J. M.; Fan, Y. N.; Zou, Z. G.; Hu, Z. J. Power Sources 2010, 195, 7578.

[24] Li, D. Q.; Zhang, Z. Q.; Zang, P. Y.; Ma, Y. W.; Wu, Q.; Yang, L. J.; Chen, Q.; Wang, X. Z.; Hu, Z. Acta Chim. Sinica 2016, 74, 587. (黎 聑勤, 张志琦, 藏鹏远, 马延文, 吴强, 杨立军, 陈强, 王喜章, 胡征, 化学学报, 2016, 74, 587.)

[25] Wang, H. S.; Wingender, C.; Baltruschat, H.; Lopez, M.; Reetz, M. T. J. Electroanal. Chem. 2001, 509, 163

[26] Mihalcik, D. J.; Lin, W. B. Angew. Chem., Int. Ed. 2008, 47, 6229.

(Zhao, X.) 